\title{
Unilateral vocal cord paralysis following high oesophageal stricture dilatation: case report and review of the literature
}

\begin{abstract}
Symptomatic vocal cord paralysis is an extremely rare complication of oesophageal dilatation or stent insertion, with only two previously reported cases described in the literature. In both cases, paralysis was suggested to be caused by recurrent laryngeal nerve compression from stent or dilatation.

We present the case of a 63year old female who developed immediate postoperative aphonia following a proximal oesophageal dilatation procedure for strictures. Flexible nasendoscopy of the larynx identified complete left sided vocal cord paresis causing her symptoms, which was presumed to be a complication of the dilatation.
\end{abstract}

Keywords: vocal cord, paralysis, esophageal, dilatation, iatrogenic
Volume 9 Issue 3 - 2017

\section{Eugene Wong, Timothy Quy Phong Do, Thomas Emslie Stewart \\ Department of Otorhinolaryngology Concord Hospital, Australia}

Correspondence: Eugene Wong Department of Otorhinolaryngology Concord Hospital Concord NSW 2139, Australia, Tel +6I433083033, Email Eugene.hl.wong@gmail.com

Received: November 05, 2017 | Published: November 29, 2017

\section{Introduction}

Vocal cord paralysis is often a debilitating syndrome that may affect speaking, breathing and swallowing, potentially causing significant reductions in quality of life. ${ }^{1}$ It can be caused by lesions at the nucleus ambiguus, supranuclear tracts, main trunk of the vagus nerve or the recurrent laryngeal nerve. Vocal cord paralysis due to recurrent laryngeal nerve injury is usually caused by extrinsic damage during its course in the neck or thorax from tumours or trauma, insult from neurotoxins or neurotoxic infections or viral illness. ${ }^{2}$

Occasionally, iatrogenic recurrent laryngeal nerve injury can occur. Although this is most commonly associated with surgery involving the thyroid, it is also a complication in surgeries involving the anterior cervical spine and cardiac surgeries. ${ }^{3}$

Vocal cord paralysis caused as a complication of proximal oesophageal dilatation is extremely rare, with only two cases described thus far in the literature. ${ }^{4,5}$ In both cases, paralysis was bilateral. We report the case of a patient who developed left sided vocal cord paralysis following a proximal oesophageal dilatation procedure.

\section{Case presentation}

A 63year old Caucasian female initially presented to our hospital Emergency department in May 2017 due to an inability to tolerate any solid oral intake with regurgitation after eating ham for lunch. She also experienced ongoing foreign body sensation and pain at the sternoclavicular notch with profuse sialorrhea.

After admission to hospital under the care of the gastroenterology team, an oesophagoscopy was performed under general anaesthetic. During this procedure, the obstructing food bolus was removed at the upper third of the oesophagus approximately $20 \mathrm{~cm}$ from the incisors. Removal of the bolus was achieved using a Roth Net. Once the food was removed, an intrinsic stricture with mild inflammation was identified which was unable to be traversed with the gastroscope. Image from gastroscopy immediately after removal of food bolus is demonstrated in Figure 1

The stricture was thought to be secondary to adjuvant radiotherapy in 2014 for bilateral breast cancer. The patient then underwent two attempts at oesophageal dilatation using a through-the-scope balloon dilator -the initial operation using a $6 \mathrm{~mm}$ balloon dilator and a second operation one week later using an $8 \mathrm{~mm}$ dilator.

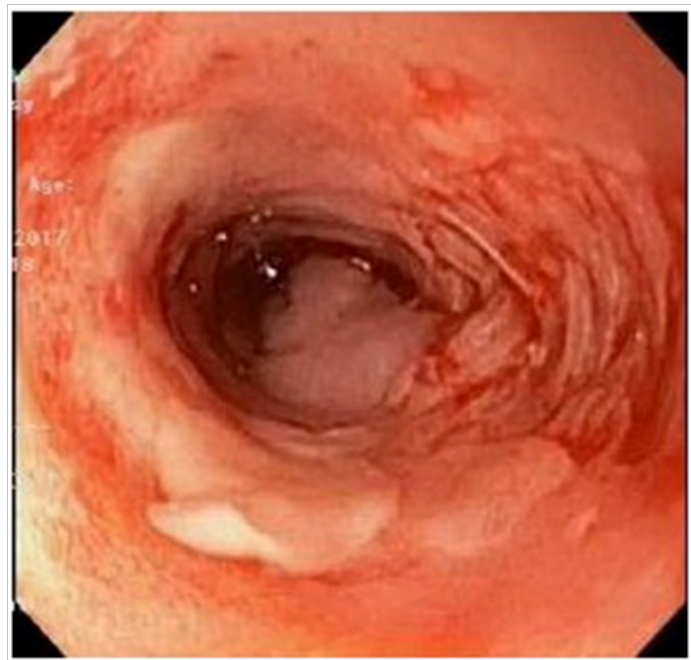

Figure I Image of the oesophagus post-dilatation on gastroscopy.

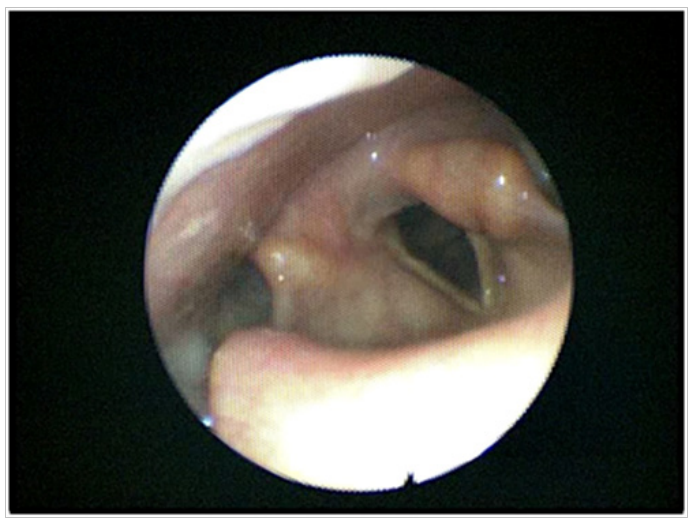

Figure 2 Image of flexible nasendoscopy of the larynx performed postprocedure demonstrating complete left sided vocal paralysis. 
Immediately postoperatively after the first dilatation, the patient experienced aphonia and was only able to speak in soft whisper. After no improvement by the second dilatation, speech pathology input was sought who suggested a barium swallow and ENT review.

Video laryngoscopy demonstrated left sided vocal cord paresis in a paramedian position without oedema or compression (Figure 2). There appeared to be incomplete compensation of the contralateral cord. The remainder of the endoscopic examination was unremarkable.

A high resolution computed tomography scan of the neck was then performed but did not demonstrate any other anatomical cause of recurrent laryngeal nerve injury. The entire course of the nerve was unremarkable bilaterally.

The presumed diagnosis at this stage was therefore of left sided recurrent laryngeal neurapraxia secondary to dilatation at the upper oesophagus. Re-examination was planned for three months after this initial video laryngoscopy to assess for recovery of nerve function, with consideration of cord bulking injection such as hyaluronic acid if paralysis did not recover by this stage.

\section{Discussion}

Vocal cord paralysis is defined as complete loss of innervation supplying motor function of the folds. The most common cause of vocal cord paralysis involves injury to the recurrent laryngeal nerve, as this directly supplies the posterior cricoarytenoid muscle that is responsible for cord abduction. Patients with vocal cord paralysis predominantly present with issues involving phonation, respiration or aspiration. ${ }^{6}$

There are many causes of vocal cord paralysis. However, there are four major causes: surgery, malignancy, idiopathic and neck trauma. Previous studies, such as those performed by Chen and Ko, have demonstrated that thyroidectomy is the most common surgical cause of vocal cord paralysis, followed by cardiac surgery. ${ }^{7,8}$ There have only been two reported cases of vocal cord paralysis following oesophageal stenting or dilatation procedures.

Gellad et al., ${ }^{4}$ described the case of an 83year old male with a history of oesophageal squamous cell carcinoma with refractory dysphagia, who developed bilateral vocal cord paralysis following dilation and stenting of a high oesophageal stricture. In this case, the patient developed inspiratory stridor hours after the procedure, which did not improve with removal of the stent, and ultimately required a tracheostomy.

Moreels et al., ${ }^{5}$ described a 56year old man who also had a history of oesophageal SCC undergoing treatment for benign postoperative stenosis of an oesophagogastric anastomosis. Balloon dilatation of the stenosis up to $8 \mathrm{~mm}$ was performed under fluoroscopic guidance, and a self-expandable metal stent was placed. The patient developed hoarseness of voice and severe stridor 4days later, and subsequently the stent was removed. This patient, however, experienced gradual recovery of cord paralysis and resolution of symptoms.

We postulate that in all three cases, injury resulted from local extrinsic compression of the recurrent laryngeal nerve as it ascends in a groove at the trachea-oesophageal junction. A study by Shao et al., ${ }^{9}$ assessing anatomical variation of the recurrent laryngeal nerve in 2404 patients found that the left nerve almost always ascended in this groove, while the right nerve tends to be much more variable in its location. This could explain why only the left vocal cord was affected in our case compared to the bilateral involvement described by Gellad and Moreels.

\section{Conclusion}

This case report highlights the need for endoscopists to be vigilant of recurrent laryngeal nerve palsy as a complication of dilatation of the upper oesophagus. We suggest that this complication be discussed with patients where loss or change in voice is a significant material risk.

\section{Acknowledgments}

This research did not receive any specific grant from funding agencies in the public, commercial, or not-for-profit sectors.

\section{Conflicts of interest}

The authors declare that there is no conflict of interest regarding the publication of this paper.

\section{Funding}

None.

\section{References}

1. Fang TJ, Li HY, Gliklich RE, et al. Quality of life measures and predictors for adults with unilateral vocal cord paralysis. Laryngoscope. 2008;118(10):1837-1841.

2. Toutounchi SJ, Eydi M, Golzari SE,et al. Vocal cord paralysis and its etiologies. J Cardiovasc Thorac Res. 2014;6(1):47-50.

3. Dankbaar JW, Pameijer FA. Vocal cord paralysis: anatomy, imaging and pathology. Insights Imaging. 2014;5(6):743-751.

4. Gellad ZF, Hampton D, Tebbit CL, et al. Bilateral vocal cord paralysis following stent placement for proximal esophageal stricture. Endoscopy. 2008; 40(Suppl 2):E150

5. Moreels TG, De Schepper HU, Macken EJ, et al. Vocal cord paralysis due to self-expandable metal stent in proximal esophagus. Endoscopy. 2014;46(S 01):E155-E156.

6. Stager SV. Vocal cord paresis: etiology, clinical diagnosis and clinical management. Curr Opin Otolaryngol Head Neck Surg. 2014;22(6):444-449.

7. Chen $\mathrm{HC}$, Jen $\mathrm{YM}$, Wang $\mathrm{CH}$, et al. Etiology of vocal cord paralysis. ORL J Otorhinolaryngol Relat Spec. 2007;69(3):167-71.

8. Ko HC, Lee LA, Li HY, et al. Etiologic Features in patients with Unilateral Vocal Cord Paralysis in Taiwan. Chang Gung Med J. 2009;32(3):290-296.

9. Shao T, Qui W, Yang W. Anatomical variations of the recurrent laryngeal nerve in Chinese patients: a prospective study of 2,404 patients. Sci Rep. $2016 ; 6: 25475$. 